\title{
A NOTE ON GROUPS WITH RELATIVELY COMPACT CONJUGACY CLASSES
}

\author{
THEODORE W. WILCOX
}

\begin{abstract}
In a more general form, the following structure theorem is proved. Let $G$ be a locally compact group with small invariant neighborhoods. Then $G$ has relatively compact conjugacy classes if and only if $G$ is a direct product of a vector group $V$ and a group $L$ where $L$ has a compact open normal subgroup $K$ such that $L / K$ has finite conjugacy classes.
\end{abstract}

The purpose of this note is to prove the following theorem which is a direct generalization of the basic structure theorem for locally compact abelian groups [2, Theorem 24.30].

THEOREM. Let $\mathscr{B}$ be a subgroup of $\mathfrak{A}(G)$ containing the inner automorphisms. Let $G \in[S I N]_{\mathscr{B}}$. Then $G \in[F C]_{\mathscr{B}}^{-}$if and only if $G$ contains $\mathscr{B}$-invariant subgroups $V, L$ and $K$ such that $V$ is a vector group, $K$ is compact and open in $L, L / K \in[F C]_{-s}^{-}$, and $G=V L$ is a direct product of $V$ and $L$.

First we establish a few definitions and some notation. All groups considered are Hausdorff and locally compact. The group operation is multiplication. A vector group is one which is topologically isomorphic to the additive structure of $R^{n}$ with $n \geqq 0$. The connected component of the identity of a topological group $G$ is denoted $G_{e}$. An element of $G$ is said to be compact if the subgroup it generates has compact closure. The group of topological automorphisms of $G$ is $\mathscr{U}(G)$. If $\mathscr{B}$ is a subgroup of $\mathfrak{U}(G)$ which contains the inner automorphisms, then the $\mathscr{B}$-orbit of $x \in G$ is $\{\beta(x): \beta \in \mathscr{B}\}$. A subset $S$ of $G$ is said to be $\mathscr{B}$-invariant, if $\beta(s) \in S$ for all $s \in S$ and $\beta \in \mathscr{B}$. We are interested in the following classes of groups.

$G \in[F C]_{\mathscr{B}}^{-}$if the $\mathscr{B}$-orbits of points have compact closures.

$G \in[S I N]_{\mathscr{B}}$ if there is a neighborhood basis of compact $\mathscr{B}$-invariant neighborhoods at the identity.

$G \in[F D]_{\mathscr{B}}^{-}$if the $\mathscr{B}$-commutator subgroup, which is the closure of the group generated by $\left\{x^{-1} \beta(x): \beta \in \mathscr{B}, x \in G\right\}$, is compact.

Received by the editors November 27, 1972.

AMS (MOS) subject classifications (1970). Primary 22D05; Secondary 43A20.

Key words and phrases. Locally compact group, splitting theorem, relatively compact conjugacy class, small invariant neighborhoods.

(c) American Mathematical Society 1974 
If $\mathscr{B}$ actually equals the inner automorphism group, then the subscript and prefix $\mathscr{B}$ is omitted. If $H$ is a $\mathscr{B}$-invariant subgroup of $G$, then the restriction of $\mathscr{B}$ to $H$ is a subgroup of $\mathfrak{U}(H)$ which, by abuse of notation, is again denoted $\mathscr{B}$. Similar remarks apply to quotients formed by $\mathscr{B}$ invariant subgroups.

The proof of the theorem relies on the following results of Grosser and Moskowitz and on the lemma below. Let $\mathscr{B}$ be a subgroup of $\mathfrak{A}(G)$ containing the inner automorphisms; let $G \in[F C]_{\mathscr{B}}^{-}$and let $P$ be the periodic subgroup of $G$, that is, $P$ is the set of compact elements of $G$.

(1) $P$ is a closed $\mathscr{B}$-invariant subgroup of $G$ and the sequence $1 \rightarrow P \rightarrow G \rightarrow W \times D \rightarrow 1$ is exact. Here $W$ is a vector grcup and $D$ is a discrete torsion-free abelian group [3, Theorem 3.16].

(2) If $G$ is compactly generated, then $P$ is compact [3, Theorem 3.20].

(3) If $G \in[F D]^{-}$, then normal vector subgroups split [3, Corollary 4.3].

LEMMA. Let $\mathscr{B}$ be a subgroup of $\mathfrak{U}(G)$ containing the inner automorphisms and let $G \in[F C]_{-3}^{-}$. If the connected component of the identity $G_{e}=V K$ is a direct product of a nontrivial $\mathscr{B}$-invariant vector subgroup $V$ and a compact group $K$, then there is a $\mathscr{B}$-invariant subgroup $L$ such that $G=V L$ is a direct product of $V$ and $L$ with $L_{e}$ compact.

Proof. Let $P$ be the set of compact elements of $G$. We claim the map $\psi: V P / P \rightarrow V /(V \cap P)=V$ defined by $\psi(v P)=v$ is a topological isomorphism. Since $V$ is $\sigma$-compact and $P$ is closed, this follows from [2, Theorem 5.33] providing we show that $V P$ is open, hence closed in $G$. Let $H$ be any compactly generated open subgroup of $G$. Then $H \in[F C]^{-}$and so by (2) $H \in[F D]^{-}$. Furthermore, $V$ is a normal vector subgroup of $H$ so that, by (3), $H=V M$ is a direct product of $V$ with a subgroup $M$. Since $H=$ $V M \supset G_{e}=V K$ and $V$ contains no compact elements, $M \supset K$. Furthermore, $M / K \cong H / G_{e}$ is totally disconnected. Thus $M$ contains a compact open subgroup $M_{1}$. Since $V M_{1} \subset V P$ and $V M_{1}$ is an open subset of $G, V P$ is open in $G$.

By (1) $G / P=W D$ is a direct product of a vector subgroup $W$ with a discrete subgroup $D$. Let $\pi_{1}: G \rightarrow G / P$ and $\pi_{2}: W D \rightarrow W$ be the canonical projections. Next note that $\pi_{1}^{-1}\left(\pi_{1}\left(G_{e}\right)\right)=G_{e} P=V K P=V P$ is open implies that $\pi_{1}\left(G_{e}\right)$ is open and hence closed and so $\pi_{1}\left(G_{e}\right)=V P / P=(G / P)_{e}=W$ [2, Theorem 7.12]. That is, $W=V P / P$. Now consider the composition

$$
G \stackrel{\pi_{1}}{\longrightarrow} G ! P=(V P / P) D \stackrel{\pi_{2}}{\longrightarrow} V P / P \stackrel{v}{\longrightarrow} V .
$$

If $v \in V$, then $\psi\left(\pi_{2}\left(\pi_{1}(v)\right)\right)=\psi\left(\pi_{2}(v P)\right)=\psi(v P)=v$. Thus $\pi=\psi \circ \pi_{2} \circ \pi_{1}$ is a projection onto the normal subgroup $V$ and $G=V L$ is a direct product with $L=\operatorname{ker} \pi$. 
We now show that $L$ is $\mathscr{B}$-invariant. Let $x \in L$ and let $O(x)$ be the closure of the $\mathscr{B}$-orbit of $x$ which is compact and $\mathscr{B}$-invariant. Let $G_{x}$ be the subgroup of $G$ generated by $O(x)$. Then $G_{x}$ is $\mathscr{B}$-invariant and so is a compactly generated $[F C]_{3}$-group. By (2) $G_{x} \in[F D]_{3}$. This means that the $\mathscr{B}$-commutator subgroup of $G_{x}$ is compact so that its image in $V$ under $\pi$ is a compact, hence trivial, subgroup of $V$. It follows that $x^{-1} \beta(x) \in$ ker $\pi=L$ and $\beta(x) \in x L=L$, for each $\beta \in \mathscr{B}$. Since $x$ was an arbitrary element of $L, L$ is $\mathscr{B}$-invariant. Since $L_{e}=(G / V)_{e}=G_{e} / V=K, L_{e}$ is compact $[2,7.13]$.

Proof of the Theorem. Assume $G \in[F C]_{\mathscr{B}}^{-} \cap[S I N]_{\not \supset \not}$. Then

$$
G_{e} \in[F C]_{\check{S}}^{-} \cap[S I N]_{\mathscr{B}}
$$

so that the closure of $\mathscr{B}$ as a subgroup of $\mathfrak{U}\left(G_{e}\right)$ is compact [3, Theorem 0.1 ]. Since $G_{e}$ is a connected [SIN]-group, it is maximally almost periodic and is a direct product $G_{e}=V_{1} K_{1}$ of a vector group $V_{1}$ and a compact group $K_{1}$ [1, Théorème 16.4.6]. Since $K_{1}$ is a characteristic subgroup of $G$, there is an automorphism $\propto$ of $G_{e}$ such that $V=x\left(V_{1}\right)$ is a $\not h$-invariant subgroup of $G$ and $G_{e}=V K_{1}$ [3, Theorem 1.1]. The lemma now applies and we have $G=V L$ with the desired properties. All that remains is to exhibit the required compact open subgroup $K$ of $L$. The totally disconnected group $L / L_{e}$ is in [SIN] $]_{\text {s }}$ so that any compact open subgroup $K_{2}$ in $L / L_{e}$ contains a $\mathscr{B}$-invariant neighborhood of the identity. Thus $\bigcap\left\{\beta K_{2}: \beta \in \mathscr{B}\right\}$ is a compact open $\mathscr{B}$-invariant subgroup of $L / L_{\varepsilon}$. Let $K$ be its inverse image in $L$.

Conversely, assume $G=V L$ as in the statement of the theorem. It suffices to show that $L \in[F C]_{\mathscr{B}}$. Let $\left\{x_{\alpha} K: x \in A\right\}$ be a coset decomposition of the discrete group $L / K$. Let $x \in L$ so that $x=x_{x} k$ for some $\alpha$. The $\mathscr{B}$-orbit $O$ of $x_{\alpha} K$ is finite. Thus, if $\pi$ is the projection of $L$ on $L / K$, we have $\pi\left(\beta\left(x_{\alpha}\right)\right) \in O$. Consequently, $\beta(x)=\beta\left(x_{\alpha}\right) \beta(k) \in \pi^{-1}(O) K$. That is, the $\mathscr{B}$-orbit of $x$ is contained in a compact subset of $L$.

REMARKS. The theorem stated above is a generalization of a structure theorem of Grosser and Moskowitz [3, Theorem 4.6]. In their case the group $G$ was in $[F D]_{3}^{-}$and they were able to choose the compact subgroup $K$ so that $L / K$ was $\mathscr{B}$-fixed. That this is not generally possible for $G \in$ $[F C]_{\bar{B}}^{-}$is illustrated by considering a group $G$ which is a discretely topologized weak direct sum of an infinite number of copies of a finite simple group [3, p. 39]. This group has finite conjugacy classes and the existence of such a (finite) subgroup $K$ would imply that $G$ had a finite commutator subgroup, which it does not.

Compactly generated locally compact abelian groups split as a direct product $R^{n} \times Z^{m} \times K$, with $K$ compact [2, Theorem 9.8]. This theorem does not generalize to any reasonable class of nonabelian groups. However, 
if we assume that $G$ is a compactly generated group in $[S I N]_{\mathscr{B}}$, the theorem remains valid with " $L / K \cong Z^{m}$ for some $m \geqq 0$ and $L / K$ is $\mathscr{B}$ fixed" replacing " $L / K \in[F C]_{\mathscr{B}}^{-}$". This can be obtained as a corollary of our theorem by utilizing the method of proof of [3, Proposition 4.5] as outlined below. Without loss of generality, we can now assume that $K$ contains the $\mathscr{B}$-commutator subgroup of $L$ so that $L / K$ is a finitely generated abelian group and then enlarge $K$ so that $L / K$ is torsion-free and $K$ is compact.

Our theorem has found applications in harmonic analysis. See [4].

The author would like to express his gratitude for the generous support of the research leading to this paper by the Technische Universität München and to his colleagues there for stimulating discussions.

\section{REFERENCES}

1. J. Dixmier, Les $C^{*}$-algebres et leurs représentations, Cahiers Scientifiques, fasc. 29, Gauthier-Villars, Paris, 1964. MR 30 \#1404.

2. E. Hewitt and K. Ross, Abstract harmonic analysis. Vol. I. Structure of topological groups. Integration theory, group representations, Die Grundlehren der math. Wissenschaften, Band 115, Academic Press, New York; Springer-Verlag, Berlin, 1963. MR 28 \#158.

3. S. Grosser and M. Moskowitz, Compactness conditions in topological groups, J. Reine Angew. Math. 246 (1971), 1-40. MR 44 \#1766.

4. E. Kaniuth and D. Steiner, On complete regularity of group algebras, Math. Ann. (to appear).

Department of Mathematics, State University College at Brockport, BrockPORT, NEW YORK 14420 